\title{
Disiscrivere e disiscriversi. oggi si può
}

\author{
Luisa di Valvasone
}

PUBBLICATO: 31 DICEMBRE 2019

I verbi iscrivere 'registrare in un elenco, lista, registro e simili' e iscriversi, nel senso di 'farsi socio di un partito, società, organizzazione e simili', sono attestati in italiano a partire rispettivamente dal I342 e dal 1908 (cfr. DELI) e mantengono oggi vitalità sia nell'uso comune sia in ambito formale e burocratico. Disiscrivere e il riflessivo disiscriversi, segnalati dai nostri lettori nel significato di 'revocare la propria o l'altrui iscrizione', sembrano essere entrati nell'uso in tempi più recenti. Sia iscrivere sia disiscrivere sono maggiormente usati alla forma riflessiva, come spesso accade per i verbi transitivi.

Per quanto riguarda la diffusione di disiscrivere possiamo rintracciare in rete diverse occorrenze. Il 4/II/20Ig tra le pagine in italiano di Google, ricercando le sole forme degli infiniti, si trovano: 8.850 risultati per disiscrivere e 9I.200 risultati per disiscriversi. Presenti anche occorrenze delle forme flesse: 5.930 risultati per disiscrivo, 4.480 per disiscrivi, 776 per disiscrivono, 259 per disiscriveró, 47 per disiscrissi. Discretamente attestato il participio disiscritto con 8.970 risultati, talvolta usato con valore aggettivale (5.980 occorrenze per il plurale: frequente la locuzione utenti disiscritti). Significativamente minori sono le occorrenze trovate su Google Libri (34 risultati per disiscrivere, Io per disiscriversi) e sugli archivi di quotidiani come "La Repubblica" (I risultati per disiscrivere, 9 per disiscriversi) e "Il Corriere della Sera" (nessun risultato per entrambe le forme).

In rete possiamo inoltre trovare alcuni esempi delle forme disinscrivere ( 375 occorrenze), disinscriversi (I48), disinscritto (274), da inscrivere, impiegate nello stesso significato. In effetti, fino al Novecento era normale l'oscillazione tra iscrivere e inscrivere (che condividono lo stesso etimo latino inscribere 'scrivere sopra'; cfr. Bruno Migliorini, Ignazio Baldelli, Breve storia della lingua italiana, Firenze, Sansoni, 1964); successivamente è avvenuta una differenziazione di significati - 'includere qualcosa o qualcuno in una lista, un elenco' e 'scrivere o disegnare qualcosa dentro una figura o sopra una superficie' (Vocabolario Treccani online) - ma ancora oggi si trovano relitti di inscrivere nel senso di iscrivere. Tornando alle nostre forme, ad oggi non se ne trovano tracce nei dizionari, con l'unica eccezione dello Zingarelli che, a partire dall'edizione 2016, pone a lemma disiscrivere; riportiamo qui l'intera voce:

disiscrivere [comp. di dis- e iscrivere - 1999] A) v. tr. - togliere dall'elenco degli iscritti il nome di qlcu. o qlco.: d. uno studente, una squadra B) disiscriversi v.rifl. - revocare la propria iscrizione a qlco.: disiscriversi da una newsletter.

Come si legge nella trattazione del lemma, disiscrivere, e ovviamente il riflessivo disiscriversi, sono composti formati dal verbo iscrivere/iscriversi con l'aggiunta del prefisso verbale dis- (formazione analoga al già trattato disassociare). Nel nostro sistema linguistico dis- può avere valore negativo, come nei verbi disapprovare, ovvero 'non approvare', dischiudere e dispiacere (cfr. Serianni 1989), o sottrattivo, come nel caso didisaggregare, disincentivare e dei nostri disiscrivere e disiscriversi; questi infatti indicano propriamente l'azione di 'cancellare il proprio (o l'altrui) nome dall'elenco degli iscritti'.

Lo Zingarelli indica come data di prima attestazione il 1999 senza riportare la fonte. Su Google Libri possiamo ritrovare unoccorrenza della forma riflessiva in una guida alla ricerca bibliografica rivolta agli studenti universitari pubblicata proprio nel I999: 
Data la vastità e varietà degli argomenti, ogni mailing list si caratterizza per una sua fisionomia [...]. Se, nell'insieme, non si è soddisfatti dell'andamento del dibattito, è molto semplice disiscriversi - senza bisogno di alcuna spiegazione - mandando un altro messaggio standardizzato (unsubscribe 'lista'). (Loretta De Franceschi, Giorgio Montechi, Libri, reti, biblioteche: guida alla ricerca, Ferrara, Tecomproject, 1999)

Da questo esempio riscontriamo innanzitutto la presenza dell'equivalente inglese unsubscribe, del quale emergono tra le pagine in italiano di Google ben I24.000.0oo di occorrenze. Si nota inoltre che disiscriversi è qui riferito all'azione di revocare la propria iscrizione a una mailing list. Dalle occorrenze che abbiamo trovato si evince che i "luoghi" da cui è possibile disiscrivere sé stessi o altri sono svariati. Ad esempio, le attestazioni trovate nell'archivio della "Repubblica", a partire dal 20I4, sono per la quasi totalità riferite alla scelta da parte di alcuni genitori di disiscrivere (talvolta scritto col trattino dis-iscrivere) i propri figli dal servizio della mensa scolastica, nel significato di 'revocare l'iscrizione al servizio mensa':

«HO dis-iscritto mio figlio dalla mensa il I4 gennaio 20r6: ora però sarei perseguibile?» [...]. A convincere Bajardo a dis-iscrivere il figlio dal servizio, a gennaio, è stato il ritrovamento di un frammento di cutter nell'insalata distribuita alla mensa della scuola [...]. (Michela Bompani, Un papá: 'Non saró più un fuorilegge del refettorio', La Repubblica.it, 6/9/2016)

Il dibattito intorno alla possibilità di disiscrivere gli studenti dalla mensa scolastica ha avuto, tra il 2014 e il 2017, un discreto riscontro nei media italiani ed è probabile che tale risonanza abbia contribuito alla scelta dello Zingarelli di mettere a lemma il verbo (e, come vedremo in seguito, anche il sostantivo derivato disiscrizione). Tuttavia in rete le occorrenze sono perlopiù relative al disiscrivere sé stessi o altri utenti (che diventano utenti disiscritti) da una mailing list o da una newsletter; in questi contesti disiscrivere e disiscriversi sono talvolta usati in alternativa a cancellare e cancellarsi sebbene, come si legge nel brano seguente, cancellare e disiscrivere non siano pienamente sinonimi:

Chiariamo infine una distinzione fondamentale: disiscrizione e cancellazione non sono sinonimi quando si parla di email marketing. Quando l'utente sceglie di disiscriversi, il suo contatto rimane a tutti gli effetti all'interno del database, ma viene spostato dall'elenco Iscritti all'elenco Disiscritti e smette di conseguenza di ricevere comunicazioni. (Maria Giulia Ganassini, Link di disiscrizione nelle email: una questione di trasparenza, reputazione, performance e deliverability, dal blog "MailUp", 23/10/2016)

Quando ti iscrivi a una lista, conserva il messaggio di conferma. Oltre a contenere le linee guida, molto probabilmente comprende anche le informazioni per iscriversi e disiscriversi e il luogo dove si può trovare l'archivio della lista, se esiste. (Gianni Lombardi, Come si fa a comunicare con la posta elettronica, Milano, Tecniche Nuove, 2006)

Nell'informativa deve essere chiaro che ci si può sempre disiscrivere dalla newsletter utilizzando l'apposito link in fondo alla mail. (Bruno Saetta, Mailchimp e GDPR, dal sito wwwprotezionedatipersonali.it, $19 / 8 / 2018$ )

Non solo è possibile disiscriversi da newsletter e mailing list, ma per estensione anche da siti, forum, social network e in generale da qualsiasi piattaforma online che richieda una registrazione e quindi un'iscrizione attraverso l'inserimento di dati:

Inoltre, all'inizio del 2010 Facebook aveva subito alcune gravi violazioni della sicurezza. Ben presto è sorto un movimento per chiedere agli amici di disiscriversi da Facebook in segno di protesta. (Siva Vaidhyanathan, La grande G: come Google domina il mondo e perché dovremmo preoccuparci, traduzione dall'inglese di Ilaria Katerinov, Firenze, Rizzoli Etas, 20I2) 
In definitiva, come suggerisce la morfologia stessa, è possibile disiscrivere o disisiscriversi da qualsiasi cosa che al tempo stesso preveda un'iscrizione, che si tratti di una lista di indirizzi email, di un servizio pubblico, di un movimento politico o di un corso universitario:

Ci si può disiscrivere dalla SIAE e che conseguenze si avrebbero? (SIAE - Conseguenze del disiscriversi, commento sul forum del sito www.pianoconcerto.it, 6/4/20I2)

Il divorzio più recente è in Calabria, a Crotone, dove c'è l'ennesima lista che non è stata certificata e così una trentina di attivisti del meetup "Gruppo storico di Crotone" dopo mesi di richieste e mail inviate a Milano e al ditettorio [sic] si sono disiscritti, e quindi usciti, dal $\mathrm{M}_{5} \mathrm{~S}$ con una raccomandata diretta allo staff di Milano. (Stefania Piras, Roma, non c'è Beppe Grillo con Virginia Raggi: nascono i mini direttori territoriali, "Il Messaggero.it", 3/6/2016)

In caso un Presidente desideri far co-gestire la squadra a un altro Presidente, quest'ultimo dovrà disiscrivere la propria squadra da Gruntopoli: per il completamento della Serie abbandonata si utilizzano i criteri di ripescaggio. (Regolamento Gruntopoli 2019/2020, dal sito www.calciopadovargio.com, $15 / 9 / 2018)$

Per disiscriversi da un corso in Moodle (perché ci si è iscritti per errore, oppure perché alla sua conclusione, superato l'esame, non si desidera più ritrovarlo nella propria pagina, ecc.) si deve cliccare su "Disiscrivimi da" nel blocco "Amministrazione del Corso" che appare in basso a sinistra in ogni corso. (dal sito dell'Università degli Studi di Padova, sezione "Guida all'uso di Moodle")

A fianco alla forma verbale si è formato il sostantivo femminile disiscrizione; quest'ultimo indica 'l'atto di disiscrivere o disiscriversi', analogamente a iscrizione 'l'atto di iscrivere o iscriversi'. Anche in questo caso, l'unico dizionario che ne registra l'uso, attestato a partire dal I999, è lo Zingarelli dall'edizione 20I6:

disiscrizione [1999] s.f. - il disiscrivere, il disiscriversi | atto formale con cui si revoca la propria iscrizione a qlco.

Le occorrenze in rete sono considerevoli (I48.ooo risultati tra le pagine in italiano di Google, I4.ooo per il plurale disiscrizioni, r.99o per la variante disinscrizione) e, cosi come il verbo, anche il sostantivo è usato sia in ambito burocratico o formale sia, nella maggior parte dei casi, in riferimento alla cancellazione da newsletter, siti web, social network e simili:

Repertorio di liste di discussione ricercabili per argomento. Per ogni lista sono fornite le istruzioni su iscrizione, disiscrizione e persona da contattare. (Accademie e biblioteche d'Italia, volume 67, Roma, 1999)

"Ho fatto richiesta di disiscrizione dal movimento chè diventato una proprietà privata, che ha introdotto dentro le liste gente arrivista di ogni classe", accusa un attivista. (Alessandra Ziniti, Il grande patto per i collegi a parlamentarie ancora aperte, La Repubblica.it, I9/r/2018)

A Genova la disaffezione per la mensa a inizio anno scolastico contava un centinaio di disiscrizioni al servizio, arrivate, ad oggi, a 357 (Mensa scolastica: chi sale e chi scende, dal sito www.foodinsider.it, 8/4/2018)

Se le e-mail inviate aggiungono valore, forniscono informazioni e sono effettivamente utili per il pubblico, i tassi di apertura aumenteranno e si ridurranno le disiscrizioni dalla mailing list. (E-mail Marketing: conosci già i 5 miti da sfatare?, dal sito www.psicologidigitali.it, r9/9/2019) 
Dagli esempi visti finora si nota una certa oscillazione per quanto riguarda la reggenza preposizionale, ma le ricerche su Google confermano una netta preponderanza di da rispetto ad a: 2.230 risultati per disiscriversi da contro 247 per disiscriversi a; 4I3 risultati per disisiscrivere da contro 34 per disiscrivere a; I2.6oo risultati per disiscrizione da contro II3 per disiscrizione a. La scelta di a in luogo di da potrebbe essere imputabile alla reggenza di iscrivere/iscriversi.

L'atto di rimuovere i dati di qualcuno o di sé stessi da una lista o da un registro al quale ci si è precedentemente iscritti è piuttosto comune e risale - se non come parola certamente come azione - a ben prima che le newsletter e i siti web diventassero una presenza quotidiana nelle vite di tutti. Anche precedentemente all'avvento di internet era possibile, ad esempio, iscriversi e poi cancellare la propria iscrizione alluniversità, a un ordine professionale o a un partito politico. È probabile che la formazione del verbo sia stata ritardata per motivi di eufonia: disiscrivere, con due $s$ e $3 i$ ravvicinate, poteva sembrare cacofonico. Il rapido diffondersi di mailing list, siti, forum, social media che richiedono di norma l'iscrizione (accompagnato dall'informatizzazione, ad esempio, di registri nazionali o di procedure per l'iscrizione a servizi pubblici) potrebbe dunque aver contribuito a far emergere una lacuna nel lessico italiano e conseguentemente la necessità di colmarla con una parola che definisse un'azione diventata comune, per non dire quotidiana. Regolare dunque, oltre che in linea con i normali processi morfologici dell'italiano, lo sviluppo da iscrivere a disiscrivere e, naturalmente, da iscriversi a disiscriversi; la maggior frequenza della forma riflessiva è data dal fatto che le procedure per inserire o cancellare i propri dati da un sito o una mailing list sono solitamente eseguibili ed eseguite dall'utente stesso.

È bene notare infine che i termini iscrivere e iscrizione sono molto comuni e da lungo tempo presenti nella nostra lingua, anche in ambiti settoriali, dal burocratese al linguaggio matematico o in polirematiche (ovvero locuzioni dal significato unitario non desumibile da quello delle singole parole che le compongono) come iscrizione a ruolo, iscrizione in curva, libretto discrizione (cfr. GRADIT). La "forza" di queste parole ha reso lineare il processo di formazione dei nostri disiscrivere e disiscriversi, e ha impedito agli equivalenti inglesi frequenti anche nei siti italiani, subscribe e unsubscribe (i quali tuttavia mantengono nella lingua d'origine anche il primo significato di 'sottoscrivere un abbonamento a pagamento'), di penetrare nel nostrano lessico del web. Questo è certamente un dato positivo e un segno di vitalità della nostra lingua giacché, come sappiamo, accade sempre più spesso, e particolarmente nel linguaggio del web, che l'italiano prenda in prestito anglismi, in molti casi non adattati, anche quando ciò si potrebbe evitare ricorrendo a traducenti italiani (si vedano ad esempio alcune parole nuove trattate dalla nostra redazione).

\section{Cita come:}

Luisa di Valvasone, Disiscrivere e disiscriversi: oggi si può , "Italiano digitale", 2019, XI, 2019/4 (ottobre-dicembre)

DOI: $10.35948 / 2532-9006 / 2020.3267$

Copyright 2019 Accademia della Crusca

Pubblicato con licenza creative commons CC BY-NC-ND 